\title{
Experimental Evaluation of a Routing Protocol for Wireless Sensor Networks: RPL under study
}

\author{
Karel Heurtefeux \\ Qatar Mobility Innovations Center \\ Doha, Qatar \\ Email: karelh@qmic.com
}

\author{
Hamid Menouar \\ Qatar Mobility Innovations Center \\ Doha, Qatar \\ Email: hamidm@qmic.com
}

\begin{abstract}
In this article, experimental results on the Routing Protocol for Low-Power and Lossy Networks (RPL) are presented. We study the RPL properties in terms of delivery ratio, control packet overhead and dynamicity. The results are obtained by several experimentations conducted in a large wireless sensor network testbed composed of more than 250 sensor nodes. In this real-life scenario (high density and convergcast traffic), several intrinsic characteristics of RPL are underlined: path length stability but reduced delivery ratio and important overhead. To the best of our knowledge, it is the first study of RPL on a such large platform.
\end{abstract}

\section{INTRODUCTION}

Wireless Sensor Networks (WSNs) are formed by hundreds or thousands of low energy wirelessly interconnected sensor devices. With these constraints, designing routing protocols is quite challenging. Recently, WSNs have been brought into reality with the effective deployment of sensor nodes. In this context, the Internet Engineering Task Force (IETF) Routing Over Low power and Lossy networks (ROLL) working Group was formed to develop an adapted routing solution. After 4 years, RPL was adopted by the IETF in March 2012 [1].

In [2] and [3], the authors present a performance evaluations of RPL based on simulations. In [2], the simulations are performed with the help of topology and link quality data from a real sensor network. The authors investigated path quality, routing table size, control packet overhead and connectivity. They observed that the number of control packet decreases during the simulation and the stabilization of the DODAG. In [3], the authors show that RPL allows a fast network set-up but, contrary to [2], they also find that RPL has an important control packet overhead during the simulation. More recently, [4] presents the most relevant research efforts made around RPL protocol and an experimental performance evaluation of RPL. The experimentations presented are based on 30 TelosB motes, and gives a performances evaluation in terms of packet loss, packet delay, DODAG (Destination Oriented Directed Acyclic Graph) time convergence and power consumption. However, the authors have not investigated the impact of the power transmission on packet delivery ratio and network overhead.

As per our best knowledge, this is the first study evaluating the performances of RPL in real scenarios based on a real and large platform. We believe that the understanding of RPL's

This work was made possible by NPRP grant NPRP4-553-2-210 from the Qatar National Research Fund (a member of The Qatar Foundation). The statements made herein ae solely the responsibility of the authors. behavior in realistic scenarios and environment is important and worth to investigate.

The rest of this paper is organized as follows. Section II briefly describes RPL, Section III describes the WSN experimentation platform and parameters, Section IV presents and discusses the obtained results on the RPL behavior and performances, and finally Section $\mathrm{V}$ concludes this contribution and discusses potential further work directions.

\section{RPL OVERVIEW}

RPL [5] is a hierarchical, proactive and IPv6 distance vector protocol. It constructs a DODAG and the data packets are routed through it. DODAGs have the property that all edges are destination oriented in such a way that no cycles exist. Thanks to the DODAG, each node has a rank. The rank defines the node's position relative to other nodes with respect to the DODAG root. The node's rank strictly increases from the root towards the leaf nodes. The rank is computed depending on the DODAG's Objective Function (OF): hop counts, link metrics (ETX, i.e. the expected number of transmissions required to successfully transmit and acknowledge a packet on the link or LQI, i.e. the Link Quality Indicator) or other constraints. To build and maintain its logical topology (route, parents, neighbors table), RPL uses IPv6 control messages:

- DIO: DODAG Information Object (multicast). The DIO packet carries information that allows a node to discover a RPL instance, learn its configuration parameters, select a DODAG parent set, and maintain the DODAG. They are first sent by the root (or sink) and then periodically by each node of the DODAG. In absence of change in the DODAG structure, the period duration increases exponentially.

- DIS: DODAG Information Solicitation (multicast) is used when a node joins the network in order to solicit a DIO from a RPL node.

- DAO: Destination Advertisement Object (unicast). The DAO is used to propagate destination information upwards along the DODAG. The message is unicast by the child to the selected parent to advertise their addresses and prefixes. When a node receives a DAO, it updates its routing table.

Finally, RPL has been designed to deal with constraints in energy and channel capacity. As a result, to reduce the control messages overhead, RPL uses a slow proactive process to maintain a routing topology but a reactive process to resolving 
routing inconsistencies. The reader is invited to refer to [5] for more details on RPL.

\section{METHOdOLOGY AND MATERIALS}

\section{A. Platforms description}

The SensLAB platform [6] is a set of 1000 sensor nodes available as a testbed for distributed embedding sensor network applications and distributed systems research. In this study, we used a subset of 100 nodes on Lille SensLAB platform. The nodes are randomly deployed in an indoor environment. SensLAB nodes are composed of 2 WSN430 boards (one open node and one control node) connected by one gateway board. The purpose of the control node and the gateway board is to offer the essential SensLAB features: firmware deployment on open node; radio environment and power monitoring; configurable sensor polling on control node (temperature, light); remote software update ability for control nodes and gateway. In other words, each node is connected in an "out-of-band" fashion, to a node handler using testbed infrastructure. We are able to monitor a set of metrics (packet sent or received, RSSI, noise level, temperature, light or energy level) without using wireless communications nor back end data collected by a sink. The open nodes are notably composed of:

- MSP430 core (MSP430F1611, offering 48kbyte ROM, and 10kbyte RAM) ;

- $\quad$ TI CC2420: a single-chip 2.4GHz IEEE 802.15.4 compliant RF transceiver and emitting between -25 and 0 $\mathrm{dBm}(0.003$ and $1 \mathrm{~mW})$ with maximum transmission rate of $250 \mathrm{kbps}$;

- Omnidirectional PCB antenna ;

- Varta Polyflex rechargeable battery.

For more details, we invite reader to consult [6] and [7].

\section{B. Experiment parameters}

Sensor nodes use the open source operating system Contiki. Contiki is specially designed for low-power and memoryconstrained devices. Contiki contains several lightweight network mechanisms: the uIP TCP/IP stack [8], the Rime stack [9] and the uIPv6 stack [10]. In this study, the UIPv6 stack is used, which provides IPv6 networking and contains RPL routing protocol. As a MAC layer, the sensor nodes use a simple MAC layer called sicslomac for packaging radio packets into 802.15.4 frames.

Sensor nodes periodically send data packets to one specific sensor node, the sink, using RPL to reach their destination. Note that in this study, the MAC layer is not investigated. A network is able to run multiple instances of RPL concurrently. In these experiments, only one instance is running in the network. Each experiment lasts 2 hours. It represents more up to 50000 IPv6 packets exchanged for each experiment. Thanks to the "out-of-band" infrastructure, each packet sent or received is monitored. We vary the transmission power level and investigate the RPL behavior in terms of delivery ratio, control packets overhead, dynamicity and path length. Figure 1 shows our experimental testbed for the evaluation of RPL in the multi-hop topology. It is common that some of the sensor nodes couldn't participate to the routing because they are isolated (without DODAG neighbors). They do not appear in the figure.

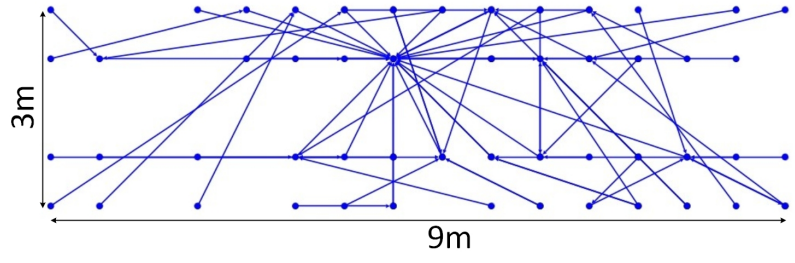

Fig. 1. RPL Logical Routing Topology with a transmission power $-25 \mathrm{dBm}$ in the Lille SensLAB platform.

Table I sums up the essential experiment parameters and platform description.

\begin{tabular}{|c|c|}
\hline & INRIA Lille \\
\hline Environment & Indoor \\
\hline Sensor Node position (3D) & Random \\
\hline Number of sensor nodes & 100 \\
\hline Radio chip & TI CC2420 \\
\hline Transmission power & $-25 \mathrm{dBm}$ to $-5 \mathrm{dBm}$ \\
\hline Frequency & $2.4 \mathrm{GHz}$ \\
\hline Experiment duration & $2 \mathrm{~h}$ \\
\hline DATA packet period & $45 \mathrm{~s}$ \\
\hline MAC protocol & sicslomac \\
\hline
\end{tabular}

TABLE I. SUMMARY OF THE EXPERIMENT PARAMETERS.

\section{EXPERIMENTAL RESULTS}

\section{A. Data Packet Delivery Ratio}

Numerous studies show poor packet delivery rates from several WSN deployments [11], [12]. The authors in [12] undertake a measurement study on a large-scale and dense sensor networks deployed in the wild, GreenOrbs. They underline that some intermediate sensor nodes bottleneck the entire network and the importance of the environment which has an unpredictable impact on the sensor network. Zhao et al. in [11] show that a large part of the link experienced more than $10 \%$ packet loss. Packet loss can be caused by many reasons: asymmetric links, fading, multipath, signal attenuation, interferences or collisions.

However, RPL has been developed especially to consider lossy networks. Despite its conception, the results show that RPL experiences an important packet loss. For instance, Figure 2 illustrates the delivery ratio obtained with different transmission power. Contrary to what we expected, the best performance is obtained with the lower transmission power. This is due to the logical routing topology based on the DODAG which create an important congestion at the sink. The logical routing topology is represented in Figure 3. The important number of 1-hop nodes can be observed in the cases a) and b). This configuration leads to a bottleneck which avoids the data messages to reach the sink. At the other hand, a lower transmission power increases the average path length (in hops) but also limits the interferences and the bottleneck effect near the sink.

Figure 4 represents the delivery ratio according the distance with a $-25 \mathrm{dBm}$ transmission power. The sensor nodes are categorized in the network according to their euclidian distance to the sink. Note that a sensor node sometimes switches its parent, resulting in dynamic routing paths to the sink of different hop counts. Consequently, we have chosen to use 


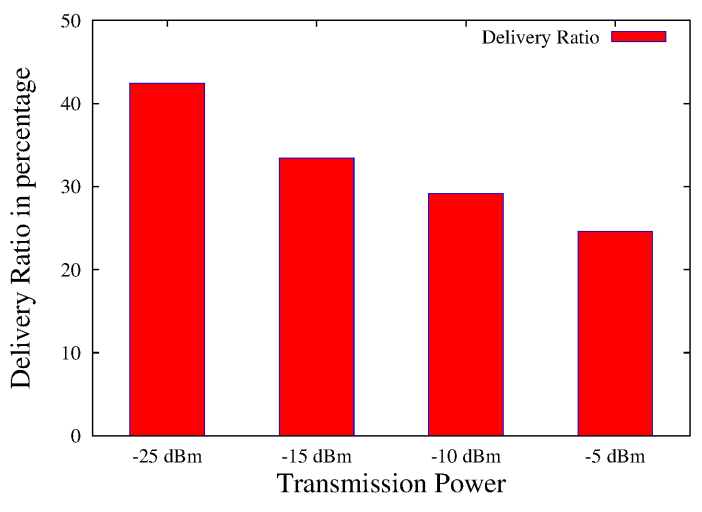

Fig. 2. Delivery ratio according transmission power
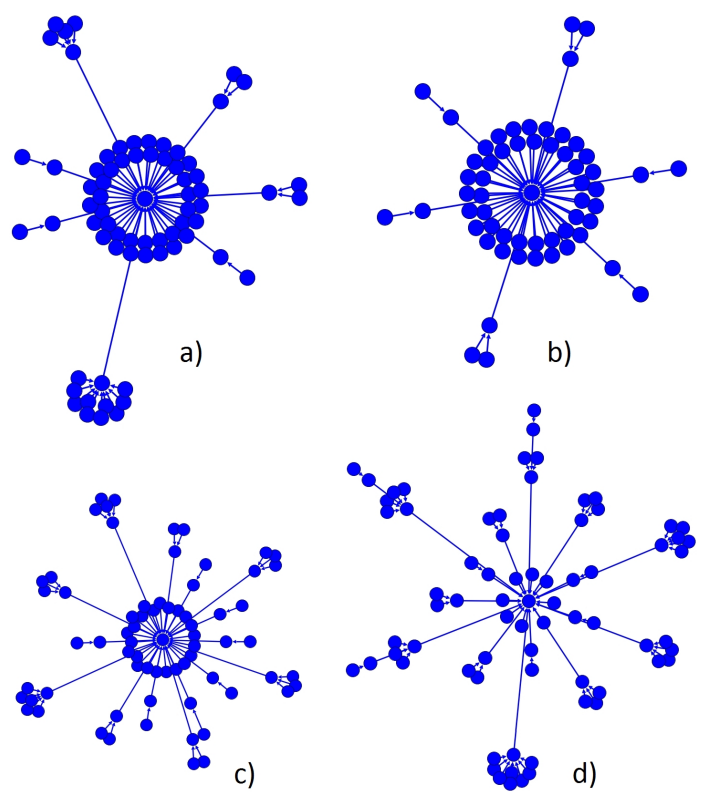

Fig. 3. RPL Logical Routing Topology with a transmission power of a) - $5 \mathrm{dBm}$; b) $-10 \mathrm{dBM}$; c) $-15 \mathrm{dBm}$ and d) $-25 \mathrm{dBm}$. For greater clarity, sensor nodes are positioned according their distance, in hops, to the sink (in center)

euclidian distance instead of hop distance. Such a result is not surprising. It is now well known that part of the data packets is lost during the forwarding process (due to packet queue overflow and collisions). Moreover, if a sensor node is distant from the sink, the path to reach this sink will be composed of links longer and weaker than the average. Such results are directly related to the choice of the Objective Functions and raise questions about the effectiveness of the default OF based on the ETX metric to select routes.

\section{B. Control Packet Overhead}

In this section, the ratio between data packets and control packets is studied. In a dynamic environment with a pro-active protocol like RPL, control packets are used to build and update the DODAG, compute the routes and maintain neighborhood and routes tables. When the network is steady, the routing topology is maintained using a low-rate beaconing process. In the other hand, if an inconsistencies is detected, the beacon rate is increased temporarily. This mechanism, governed by

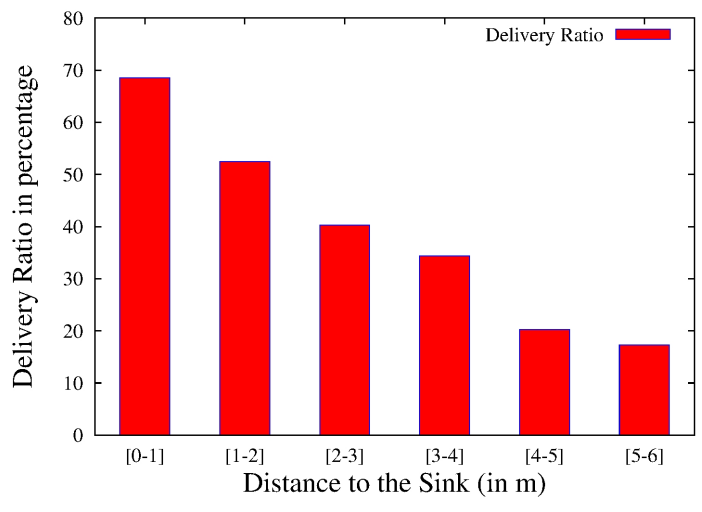

Fig. 4. Delivery ratio versus distance to the sink

Trickle timers [13], is supposed to reduce the amount of control packets while quickly resolving routing issues.

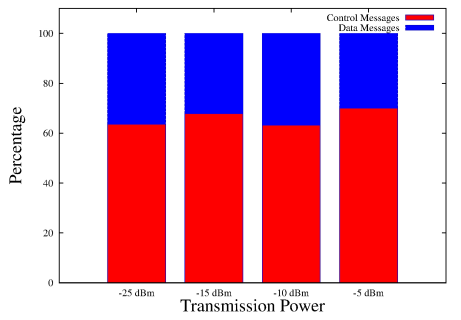

Fig. 5. Ratio between control messages and data messages with different transmission power

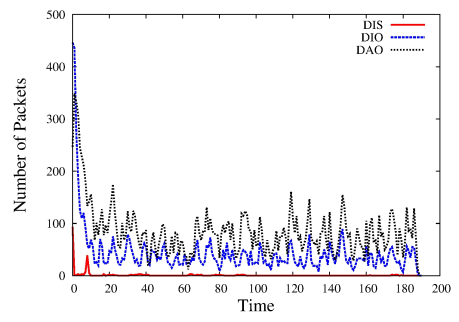

Fig. 6. RPL overhead in WSN with 100 sensor nodes and a $-25 \mathrm{dBm}$ transmission power for DIS, DIO and DAO messages
However, our results show that the amount of control messages is higher than data messages. These results differ from those obtained in [2] but are similar to those obtained in [3], both based on simulations. Figure 5 illustrates the ratio between control messages (composed of DIS, DIO and DAO messages) and data messages. Whatever the transmission power, the ratio between IPv6 control messages and data messages stays relatively stable. Among the control messages, a large part is composed of DAO messages, while DIO and DIS messages represent only $20 \%$ and less than $1 \%$ respectively. There are several reasons of this proportion. Firstly, DIS packets are used by a sensor node joining the network. As a result, DIS are sent at the very beginning of the network life and when a sensor node has been disconnected from the network (as shown in Figure 6). Figure 6 shows the overhead according the time. We see on this figure that the DIS traffic is negligible and an important part of the overhead is sent at the birth of the network and then, DIS and DIO are sent periodically. Secondly, DIO and DIS messages have the scope of a link. It means they are not forwarded while DAO message is used to propagate destination information upward along the DODAG. These results mean that a large part of energy is wasted for routing signaling. A better timers calibration is clearly required and could eventually solve this issue.

\section{Dynamicity}

Such results (low delivery rate and large overhead) are the consequence of an important dynamicity even in a static 
(without mobility) network. In WSN, the physical links are transilient and not robust. In [14], we showed that a large part of links was not symmetric or only temporarily. This phenomenon creates instability in the logical topology. Figure 7 illustrates this instability by underlining the number of neighbors update (add or remove a neighbor from the neighborhood table) per minute. The routing logical structure as well as the DODAG, are impacted by such instability because it affects parent-child connection and path metrics (hop count and ETX). As a result, the average rank, which is a combination of ETX and hop distance, may highly evolve during the experimentation (see Figure 8).

Despite the evolution of the average rank level, the average path length (i.e. the distance in number of hops between a source and the destination) remains steady. It means that the efficiency of the routing process, in other words, the ability to find the shortest paths is preserved.

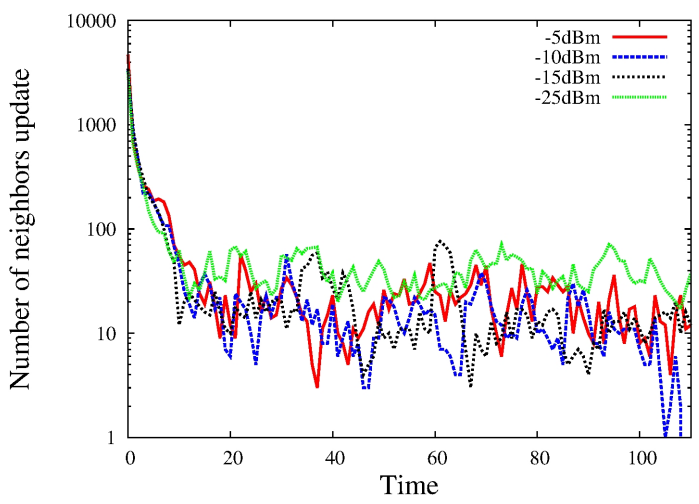

Fig. 7. Evolution of the number of update per time unit
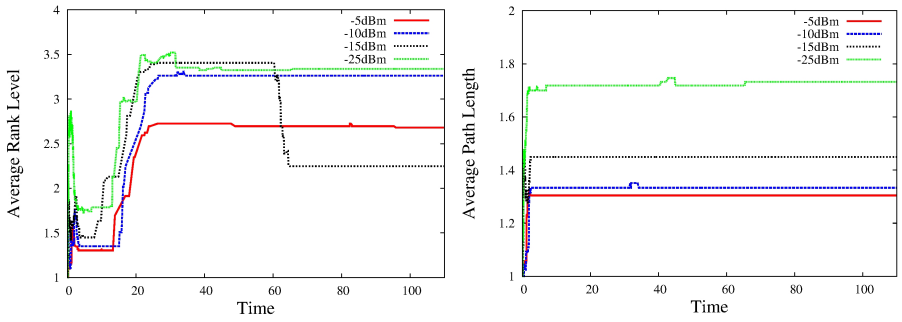

Fig. 8. Evolution of Average Rank Fig. 9. Evolution of Average Path

\section{CONCLUSion And Future Work}

We have investigated the behavior of RPL deployed in a real and dense sensor network. In particular, we have studied efficiency in terms of delivery rate, control packet ratio, and dynamicity. The main contribution in this work is to underline several key behaviors of the RPL protocol in a large and dense WSN:

- The strong stability of the path length despite the instability of the physical topology;

- The stabilization of the logical routing structure takes time and is subject to change;

- Despite the efficiency of the routing protocol to find shortest path, the delivery rate is quite low in particular for very dense networks;
- The major part of the IPv6 traffic is composed of control packets.

Finally, RPL could be considered as a smart routing protocol when considering adaptative period for control messages and efficient to build shortest path. Even in a very dense network, RPL is able to work and achieve to transmit a non negligible part of the traffic. However, to be more efficient, the Trickle mechanism should be adapted and a better calibration of the timers is clearly required. In future works, we will also investigate the impact of the objective function on the stability and the efficiency of RPL. Moreover, it would be interesting to study the impact of the sensor node outage and node addition in the DODAG evolution. In addition, the MAC layer mechanisms, not studied here, have certainly an impact on the performances of RPL and have to be investigated.

\section{REFERENCES}

[1] "Internet engineer task force," http://www.ietf.org.

[2] J. Tripathi, J. de Oliveira, and J. Vasseur, "A performance evaluation study of rpl: Routing protocol for low power and lossy networks," in Information Sciences and Systems (CISS), 2010 44th Annual Conference on, march 2010, pp. 1 -6.

[3] N. Accettura, L. Grieco, G. Boggia, and P. Camarda, "Performance analysis of the rpl routing protocol," in Mechatronics (ICM), 2011 IEEE International Conference on, april 2011, pp. 767 -772.

[4] O. Gaddour and A. KoubíA, "Survey rpl in a nutshell: A survey," Comput. Netw., vol. 56, no. 14, pp. 3163-3178, Sep. 2012. [Online]. Available: http://dx.doi.org/10.1016/j.comnet.2012.06.016

[5] A. Brandt, J. Hui, R. Kelsey, P. Levis, K. Pister, R. Struik, J. Vasseur, and R. Alexander, "Rpl: Ipv6 routing protocol for low-power and lossy networks," March 2012. [Online]. Available: http://www.rfc-editor.org/rfc/rfc6550.txt

[6] "Very large scale open wireless sensor network testbed," http://www.senslab.info, 2010.

[7] "Chipcon inc, cc2420 datasheet," http://www.ti.com/product/cc2420, 2008.

[8] A. Dunkels, "Full tcp/ip for 8-bit architectures," in Proceedings of the 1st international conference on Mobile systems, applications and services, ser. MobiSys '03. New York, NY, USA: ACM, 2003, pp. 8598. [Online]. Available: http://doi.acm.org/10.1145/1066116.1066118

[9] A. Dunkles, "Rime: A lightweight layered communication stack for sensor networks," in Proceedings of the European Conference on Wireless Sensor Networks (EWSN), Poster/Demo session, Delft, The Netherlands, jan 2007. [Online]. Available: http://dunkels.com/adam/dunkels07rime.pdf

[10] M. Durvy, J. Abeillé, P. Wetterwald, C. O'Flynn, B. Leverett, E. Gnoske, M. Vidales, G. Mulligan, N. Tsiftes, N. Finne, and A. Dunkels, "Making sensor networks ipv6 ready," in Proceedings of the 6th ACM conference on Embedded network sensor systems, ser. SenSys '08. New York, NY, USA: ACM, 2008, pp. 421-422. [Online]. Available: http://doi.acm.org/10.1145/1460412.1460483

[11] J. Zhao and R. Govindan, "Understanding packet delivery performance in dense wireless sensor networks," in Proceedings of the 1st international conference on Embedded networked sensor systems, ser. SenSys '03. New York, NY, USA: ACM, 2003, pp. 1-13. [Online]. Available: http://doi.acm.org/10.1145/958491.958493

[12] Y. Liu, Y. He, M. Li, J. Wang, K. Liu, L. Mo, W. Dong, Z. Yang, M. Xi, J. Zhao, and X.-Y. Li, "Does wireless sensor network scale? a measurement study on greenorbs," in INFOCOM, 2011 Proceedings IEEE, april 2011, pp. $873-881$.

[13] P. Levis, T. Clausen, J. Hui, O. Gnawali, and J. Ko, "The trickle algorithm," March 2011. [Online]. Available: https://tools.ietf.org/html/rfc6206

[14] K. Heurtefeux and F. Valois, "Is rssi a good choice for localization in wireless sensor network?" in Advanced Information Networking and Applications (AINA), 2012 IEEE 26th International Conference on, march 2012, pp. $732-739$. 